\title{
Study of a Population of Triplet Pregnancies: Maternal and Neonatal Outcomes
}

Donatella Caserta $^{1 *}$, Giulia Bordi ${ }^{1}$, Michele Stegagno ${ }^{1,2}$, Francesca Filippini' ${ }^{2}$ Maria Podagrosi ${ }^{2}$, Domenico Roselli ${ }^{1}$ and Massimo Moscarini ${ }^{1}$

${ }^{1}$ Department of Woman Health and Territory's Medicine, University of Rome Sapienza, S. Andrea Hospital, Rome, Italy

${ }^{2}$ Neonatal Unit of San Pietro FBF Hospital, Rome, Italy

\begin{abstract}
Objective: To describe maternal complications, perinatal mortality and neonatal morbidity in triplet pregnancies.

Methods: Retrospective analysis of maternal and neonatal records of 21 triplet pregnancies delivered from January 2008 to June 2011 at San Pietro FBF Hospital of Rome, a tertiary medical center.

Results: The incidence of triplet pregnancies was one in 690 births. Among the 21 triplet pregnancies, only one was conceived spontaneously and 20 were the result of assisted reproductive techniques. Mean maternal age was 34.4 \pm 5 years. There was one case of a selective foeticide of a foetus with an encephalocele. Preterm delivery occurred in all the remaining 20 pregnancies. Other frequent complications were premature rupture of membranes (40\%) and cervical incompetence (35\%). There was no maternal mortality in any of the studied pregnancies. The mean length of maternal hospitalization was $15 \pm 5.9$ days. The mean gestational age was 31.8 weeks, with a mean birth weight of $1590 \pm 590$ grams. The neonatal mortality was $5 \%$. The incidence of congenital anomalies was $16.7 \%$. Respiratory distress syndrome occurred in $53 \%$ of cases and $75.6 \%$ of newborns required intensive care. Other common neonatal complications were jaundice $(66.7 \%)$, anaemia $(28.9 \%)$, patent ductus arteriosus $(24.4 \%)$, apnea of prematurity $(17.8 \%)$, sepsis $(13.3 \%)$, neurological complications $(11.1 \%)$ and bronchopulmonary dysplasia $(11.1 \%)$. The mean length of newborns hospitalization was $33.6 \pm 23.3$ days. There was no significant difference in neonatal mortality and morbidity based on birth order.

Conclusions: This series of triplet pregnancies demonstrated an excellent survival rate and a relatively low major morbidity, despite the $100 \%$ incidence of prematurity. Although perinatal outcomes in triplet gestations have improved in recent years, higher-order multiple pregnancies continued to be associated with great medical problems for both mothers and infants. All methods of assisted reproduction should aim for the prevention of multifetal pregnancies.
\end{abstract}

Keywords: Triplet; Maternal outcomes; Perinatal outcomes; Assisted reproductive technology.

\section{Introduction}

The incidence of multiple gestations in developed world has increased over recent decades. In Italy, the rate of twin pregnancies and triplet and higher-order multiple pregnancies has been reported to be 12 per 1,000 and 0.52 per 1,000 respectively [1]. This increase is not limited to Italy, but it is a world-wide issue. The incidence of triplet and higher-order multiples pregnancies in the United States has increased from 0.37 per 1,000 live births in 1980 to 1.48 per 1,000 in 2008 [2].

The increase in the triplet birth rate has been most marked in women aged 40 years and over, with an increase of more than $1000 \%$. Conversely, in women aged 30 to 40 years the multiple birth rate has risen by $30 \%$ and in women aged under 20 by only $13 \%$ [3].

This steep rise is attributed to the trend to delay in the childbearing and notably to the growing use and improvement in infertility therapies and assisted reproductive techniques (ART) $[4,5]$. Over the past few years the maternal and neonatal impact of multiple pregnancies has led to a more conservative embryo transfer policy [6]. Nevertheless, ART continues to contribute to multiple birth rates, particularly to the incidence of triplet and higher-order pregnancies.

The risk of adverse short- and long-term birth outcome increases with the number of foetuses in a multigestation pregnancy. Neonatal outcome among higher-order multiples is largely determined by gestational age at birth and correlating birth weight [7]. Literature reports the mean gestational age for triplets at about 32 weeks and the rate of growth restriction at $50-60 \%$ [8].

The rate of preterm births in triplets is very different from that in twins or singletons. For example, the USA National Vital Statistics Report describes a rate of very preterm births ( $<32$ weeks of gestation) of $38.5 \%, 3.3$ times more than for twins and 24.1 times more than for singletons [2].

Multiple-pregnancy births are associated with major medical problems for both mother and infants such as preterm delivery, preeclampsia, intrauterine growth retardation, increased cesarean rates, prematurity complications, disabilities and death [9].

The socioeconomic and the emotional demands that may accompany the birth of three or more premature babies at the same time may overwhelm the mother and her family [10]. Beyond of these factors, the effects of significantly increased medical expenses on the

${ }^{*}$ Corresponding author: Donatella Caserta, University of Rome Sapienza Department of Woman Health and Territory's Medicine, S. Andrea Hospital, Via di Grottarossa 1035-1039 00189 Rome, Italy, Tel: +390633775696; Fax: +390633776660; E-mail: donatella.caserta@uniroma1.it

Received January 26, 2012; Accepted March 14, 2012; Published March 18 2012

Citation: Caserta D, Bordi G, Stegagno M, Filippini F, Podagrosi M, et al. (2012) Study of a Population of Triplet Pregnancies: Maternal and Neonatal Outcomes. J Neonatal Bio 1:104. doi:10.4172/2167-0897.1000104

Copyright: ( 2012 Caserta D, et al. This is an open-access article distributed under the terms of the Creative Commons Attribution License, which permits unrestricted use, distribution, and reproduction in any medium, provided the original author and source are credited. 
healthcare system must also be considered. While carrying a multiple pregnancy, women are more likely to generate extra medical costs due to longer period of bed-rest, hospitalization, administration of medication to prevent preterm labour and surgical procedures such as emergency caesarean section and cervical cerclage. Compared with singleton, obstetric care costs are 1.94 and 3.96 times higher for twin and triplet pregnancies, respectively. The ratio of neonatal singleton versus twins and triplets costs is $1: 16$ and 1:109 respectively [11]. Lifetime costs for chronic medical care, rehabilitation and special education related to extreme prematurity also have to be taken into account [12].

Because of decisions such as abortion, embryo reduction, life style changes and other therapeutic interventions based on these concerns, it is critical to have contemporary and accurate data on the outcomes of the neonates from multiple births [13].

The aim of our study is to evaluate maternal outcomes, perinatal mortality and neonatal morbidity in triplet pregnancies.

\section{Materials and Methods}

From January 1, 2008 to June 30, 2011, there were 14,499 deliveries at San Pietro FBF Hospital of Rome, a tertiary medical center with an University affiliated Obstetric Unit dealing with high risk cases. This study concentrated on twenty-one women with triplet pregnancy, from their antenatal care to delivery at San Pietro Hospital during above the period.

Institutional ethical committee approval was not required as this study was an observational analysis and no intervention was introduced.

The gestational age was calculated by adding two weeks to the day of oocyte retrival for assisted conception triplet pregnancies and in the one patient who spontaneously conceived, from the last menstrual period.

From the medical records of these patients, the following data were collected: age at delivery, mode of conception, type of infertility treatment, gravity and parity status, systemic diseases, gynaecologic surgery, smoking habit and weight gain during pregnancy, gestational age at the time of delivery, mode of delivery, antenatal, intrapartum and postpartum complications, antenatal admission, corticosteroids administration (defined as receipt of maternal steroid within 7 days of the delivery), antenatal admission, admission to an intensive-care unit (ICU) and length of hospitalization. The data extracted from neonatal charts included: birth weight, length, head circumference, Apgar score at 1 and 5 minutes after birth, congenital anomalies, neonatal mortality, neonatal complications, admission to neonatal intensive care unit (NICU) and length of stay in hospital. Additional information was obtained by personal communication with parents to analyze the current health status of the neonates.

Low birth weight (LBW), very low birth weight (VLBW) and extremely low birth weight (ELBW) were defined as a birth weight $<2500,<1500$ and $<1000 \mathrm{~g}$, respectively.

Antenatal corticosteroids were administrated to women at risk of iatrogenic or spontaneous preterm birth up to $34+6$ weeks of gestation, according to the guidelines of Royal College of Obstetricians and Gynaecologists (RCOG) [14].

Neonatal mortality was defined as the number of deaths within 28 days after birth per 1000 live births. Respiratory distress syndrome (RDS) was diagnosed on the basis of clinical parameters, chest radiography and blood gas values. Bronchopulmonary dysplasia was diagnosed by the need for supplemental oxygen after 28 days and the typical changes found in chest X-rays. Diagnosis of apnea of prematurity was determined when infants stopped breathing for more than 15 seconds. Ultrasound imaging diagnosed intraventricular hemorrhage (IVH). Patent ductus arteriosus (PDA) was confirmed by echocardiogram. Gastroesophageal reflux was diagnosed based on regurgitation of feeds, neck arching and posturing with feedings. Jaundice was determined by high bilirubin levels which required phototherapy. A diagnosis of anaemia was made when red cells transfusions were performed. Sepsis was diagnosed by clinical parameters and a positive blood culture. Necrotising enterocolitis was diagnosed by clinical values confirmed by radiographical pneumatosis intestinalis.

Statistics: Chi-square with Yates corrections for categorical variables and $t$-test (or non-parametric Mann-Withney-U test) for continuous variables were used to compare neonatal outcomes based on birth order. A value of $\mathrm{p}<0.05$ was considered as statistically significant.

\section{Results}

The study population included 21 women with triplet pregnancy who delivered at San Pietro FBF Hospital of Rome. The incidence of triplets was $0.145 \%$ or one in 690 of all births.

With regard to the method of conception, only one woman conceived spontaneously, whereas five pregnancies were obtained by in vitro fertilization (IVF), twelve by intacytoplasmatic sperm injection (ICSI), one by intrauterine insemination (IUI), one by gamete intrafallopian transfer (GIFT) and one by ovulation induction. All women who conceived after infertility treatments had undergone controlled ovarian stimulation.

Mean maternal age was $34.4 \pm 5$ years. All women were nulliparous, except one patient who conceived spontaneously and already had two sons. History of previous miscarriage had been reported in five patients $(23.8 \%)$ and among these, one $(4.8 \%)$ had recurrent miscarriage.

Nine women (42.9\%) had systemic diseases, including endometriosis, hypothyroidism, diabetes and thrombophilia. Eight patients (38.1\%) underwent gynaecological surgery: three patients underwent ovarian cystectomy, two myomectomy, two endometrial polypectomy and one hysteroscopic resection of uterine septum.

Only one woman (4.8\%) smoked during pregnancy. The mean weight gain during pregnancy was $16.2 \pm 8.9 \mathrm{~kg}$. Five patients $(23.8 \%)$ underwent invasive prenatal diagnosis: amniocentesis was performed in four cases and villocentesis in one case.

In one triplet pregnancy, a 40-year-old woman who conceived a triplet pregnancy after undergoing ICSI, a selective foeticide of a foetus with an encephalocele was performed. An ultrasound examination at 22 weeks of gestation showed an encephalocele in one foetus and a thick septae compatible with trichorionic triamniotic triplets. Selective foeticide was performed at 23 weeks of gestation by transamniotic injection of potassium chloride into the heart of the abnormal foetus. The procedure was without complications and the following ultrasound examinations showed that the other two twins were morphologically normal. The woman delivered at 37 weeks by elective caesarean section. The surviving twins were both boys, with an Apgar score of 8 at 1 minute and 9 at 5 minutes and weighing 2860 $\mathrm{g}$ and $2300 \mathrm{~g}$ respectively. They had no major neonatal complications 
and they were discharged 8 days after birth. Triplet 3 was a small macerated abortus.

All the remaining 20 triplet pregnancies were complicated by preterm delivery. Eight (40\%) sets of triplet were delivered before 32 weeks of gestation. The mean gestational age was $31.8 \pm 3.4$ weeks, and it ranged from 22.3 to 35.9 weeks. In addition to preterm delivery, the most common complications were premature rupture of membranes (PROM), that occurred in eight triplet pregnancies (40\%) and cervical incompetence that occurred in seven cases $(35 \%)$ and required cervical cerclage. Other complications were oligohydramnios in $30 \%$ of cases, first trimester bleeding in $25 \%$, obstetric cholestasis in $20 \%$, pregnancy-induced hypertension in $15 \%$, placental abruption in $10 \%$, gestational diabetes in $10 \%$, anaemia in $5 \%$ and hyperemesis gravidarum in $5 \%$.

Ten patients $(50 \%)$ were admitted to the antenatal unit because of pregnancy complications and prenatal corticosteroids $(12 \mathrm{mg}$ of betamethasone repeated for a total of two doses) were administrated to eighteen women (90\%).

Three patients (15\%) suffered postpartum hemorrhage which required a blood transfusion. Postpartum blood transfusion was also required in other two patients with low haemoglobin. One patient underwent peripartum hysterectomy and was transferred to intensive care unit. There was no maternal mortality in any of the studied pregnancies. The mean length of maternal hospitalization was $15 \pm$ 5.9 days.

Eighteen sets of triplet (90\%) were delivered by caesarean section. Among these, two-thirds were emergency caesareans. Two patients progressed very fast in labour at 22.3 and 26 week's gestation respectively and delivered vaginally. The leading foetus was cephalic in $12(60 \%)$ and breech in $8(40 \%)$ cases.

The percentage of male infants was $40 \%$. The mean birth weight was $1590 \pm 506$ grams. Mean birth length and head circumference were $41.7 \pm 3.9$ and $29.6 \pm 2.7 \mathrm{~cm}$ respectively. All the newborns were LBW, whereas the global rate of VLBW and ELBW was $18.3 \%$ and $16.6 \%$ respectively. Seven newborns (11.6\%) were small for gestational age (SGA) and two of them were under the 3 th percentile in weight. The remaining appropriate for gestational age (AGA) newborns was between the $10^{\text {th }}$ and $75^{\text {th }}$ percentiles in weight. No triplet was large for gestational age (LGA).

The percentage of neonates with low Apgar score $(<7)$ at 5 minutes was $30 \%$. Thirteen neonates (21.7\%) required tracheal intubation. As the triplet delivered at 22.3 weeks of gestation died shortly after birth, the neonatal mortality rate was $5 \%$. With regard to congenital anomalies, we observed ten cases (16.7\%) of birth defects, including two cases of bilateral cryptorchism, two of ventricular septal defect, four of pyelectasis and two of hypospadias.

\begin{tabular}{|l|l|l|l|l|l|l|l|l|l|}
\hline GA & $\mathbf{n}$ & Mortality & RDS & BPD & IVH & NEC & Sepsis & PDA & Jaundice \\
\hline $22-26$ & 6 & 3 & 2 & 2 & 2 & 1 & 1 & 2 & 2 \\
\hline $27-30$ & 12 & 0 & 10 & 3 & 2 & 1 & 2 & 8 & 10 \\
\hline $31-32$ & 9 & 0 & 5 & 0 & 1 & 0 & 2 & 1 & 5 \\
\hline $33-34$ & 24 & 0 & 6 & 0 & 0 & 0 & 1 & 0 & 11 \\
\hline 35 & 9 & 0 & 1 & 0 & 0 & 0 & 0 & 0 & 2 \\
\hline Total & 60 & 3 & 24 & 5 & 5 & 2 & 6 & 11 & 30 \\
\hline
\end{tabular}

GA: gestational age; RDS: respiratory distress syndrome; BPD: bronchopulmonary dysplasia; IVH: intraventricular hemorrhage; NEC: necrotizing enterocolitis; PDA: patent ductus arteriosus.

Table 1: Distribution of major neonatal complications according to gestational age.
Twelve neonates were transferred to another hospital because of capacity problems and nine of them were admitted to NICU of the new hospital because of respiratory problems. None of the transferred newborns died. Of the remaining 45 neonates, 34 (75.6\%) of them required NICU admission and 11 (24.4\%) needed for mechanical ventilation.

Table 1 depicts neonatal morbidity according to gestational age. Respiratory distress syndrome occurred in twenty-four neonates (53\%) and five newborns (11.1\%) were affected by bronchopulmonary dysplasia and needed for ventilation for more than two months. Other neonatal complications were jaundice in $66.7 \%$ of cases, metabolic acidosis in $35.6 \%$, anaemia in $28.9 \%$, patent ductus arteriosus in $24.4 \%$, apnea of prematurity in $17.8 \%$, sepsis in $13.3 \%$, hypoglycemia in $13.3 \%$, hyperglycemia in $11.1 \%$, gastroesophageal reflux in $8.9 \%$, patent foramen ovale in $6.7 \%$ and necrotizing enterocolitis in $4.4 \%$. Neurological complications were diagnosed in $11.1 \%$ of cases and included cerebral intraparenchymal or and posthemorrhagic hydrocephalus. The mean length of stay for the birth admission was $33.6 \pm 23.3$ days and it ranged from 9 to 95 days.

There was no significant difference in terms of neonatal morbidity or mortality based on birth order (Table 2). With regard to birth characteristics, mean birth weights of triplet A, B and C were $1627 \pm$ $550,1572 \pm 467$ and $1516 \pm 518$ grams respectively. Mean birth length and head circumference were 41.3 and 29.9, 42 and 29.7, 41.8 and 29.3 $\mathrm{cm}$ respectively.

\section{Discussion}

According to Hellin's rule, the natural rate for spontaneous twin

\begin{tabular}{|c|c|c|c|}
\hline Variable & Triplet A $(n=20)$ & Triplet B $(n=20)$ & Triplet C $(n=20)$ \\
\hline Mean ( $\pm \mathrm{DS}$ ) birth weight $(\mathrm{g})$ & $1572 \pm 467$ & $1526 \pm 518$ & $1627 \pm 550$ \\
\hline LBW (\%) & $20(100)$ & $20(100)$ & $20(100)$ \\
\hline VLBW (\%) & $5(25)$ & $3(15)$ & $3(15)$ \\
\hline Apgar 5'<7 (\%) & $5(25)$ & $7(35)$ & $6(30)$ \\
\hline Congenital anomalies (\%) & $4(20)$ & $4(20)$ & $2(10)$ \\
\hline Neonatal mortality (\%) & $1(5)$ & $1(5)$ & $1(5)$ \\
\hline $\operatorname{RDS}(\%)^{a}$ & $8(53.3)$ & $11(73.3)$ & $5(33.3)$ \\
\hline Apnea $(\%)^{a}$ & $4(26.7)$ & $2(13.3)$ & $2(13.3)$ \\
\hline $\begin{array}{l}\text { Bronchopulmonary dysplasia } \\
(\%)^{a}\end{array}$ & $2(13.3)$ & $0(0)$ & $3(20)$ \\
\hline $\begin{array}{l}\text { Neurologic complications } \\
(\%)^{\mathrm{a}}\end{array}$ & $1(6.7)$ & $2(13.3)$ & $2(13.3)$ \\
\hline Jaundice $(\%)^{\mathbf{a}}$ & $10(66.7)$ & $9(60)$ & $11(73.3)$ \\
\hline Anaemia $(\%)^{a}$ & $6(40)$ & $2(13.3)$ & $5(33.3)$ \\
\hline Sepsis $(\%)^{a}$ & $3(20)$ & $1(6.7)$ & $2(13.3)$ \\
\hline Necrotizing enterocolitis $(\%)^{a}$ & $2(13.3)$ & $0(0)$ & $0(0)$ \\
\hline Metabolic acidosis $(\%)^{a}$ & $5(33.3)$ & $7(46.7)$ & $4(26.7)$ \\
\hline Hypoglycemia (\%) & $1(6.7)$ & $2(13.3)$ & $3(20)$ \\
\hline Hyperglycemia $(\%)^{a}$ & $3(20)$ & $0(0)$ & $2(13.3)$ \\
\hline $\begin{array}{l}\text { Patent ductus arteriosusDA } \\
(\%)^{\mathrm{a}}\end{array}$ & $4(26.7)$ & $4(26.7)$ & $3(20)$ \\
\hline Patent foramen ovale $(\%)^{a}$ & $1(6.7)$ & $2(13.3)$ & $0(0)$ \\
\hline Gastroesophageal reflux (\%) ${ }^{a}$ & $1(6.7)$ & $1(6.7)$ & $2(13.3)$ \\
\hline NICU admission (\%) & $13(86.7)$ & $9(60)$ & $12(80)$ \\
\hline Mechanical ventilation (\%) ${ }^{a}$ & $3(20)$ & $4(26.7)$ & $4(26.7)$ \\
\hline $\begin{array}{l}\text { Length of hospitalization } \\
\text { (days) }^{\mathrm{a}}\end{array}$ & $35 \pm 23.5$ & $30.2 \pm 24.1$ & $32.3 \pm 23.7$ \\
\hline
\end{tabular}

a 12 neonates ( 4 triplet A, 4 triplets $B$ and 4 triplets $C$ ) transferred to another hospital were excluded.

Table 2: Neonatal outcomes of triplet A vs. triplet B vs. triplet $C$. 
pregnancies is $1 / 80$, the rate for triplet is $1 / 80^{2}(1 / 6,400)$ and the rate for quadruplets is $1 / 80^{3}(1 / 512,000)$ [15].

Nevertheless, the incidence of triplet pregnancies in our hospital (which represents a reference center for high-risk gestations, including multiple pregnancies and ART pregnancies) was of one in 690 deliveries $(0.145 \%)$. This notable rise is regarded mainly as a consequence of developments in assisted reproductive technologies (ART) which account for approximately $95 \%$ of triplet pregnancies [16]. This percentage is alarming and it could be explained by the attempts to increase pregnancy rates in women of further and further advanced age. Italy is one of the European countries with the highest maternal age at the first child and the lowest fertility rate [17]. The agerelated decline of the biological capacity of a woman to reproduce is well established and the current trend to postpone childbearing until later in life often requires more aggressive infertility treatments, thus increasing the risk of multiple pregnancy.

Neonatal morbidity and mortality increase with the number of foetuses in a pregnancy, but simultaneously the term of the pregnancy decreases so that problems associated with premature birth gain importance.

The incidence of preterm delivery in triplet pregnancies has been reported in literature to be approximately 90\% [2]. In accordance with other Italian research [18], all triplet pregnancies in our study population ended preterm. The optimal gestational age at birth for triplet pregnancies is reported to be 34-35 weeks, at a weight of 1,900$2,200 \mathrm{~g}$ [19]. The mean gestational age at delivery in our study was 31.8 weeks, with a mean birth weight of $1590 \mathrm{~g}$. These values are comparable with data in the literature [20-24].

Gestational age represents the main factor affecting birth weight, which correlates with morbidity and survival rate [4,25]. Alexander and colleagues [26] described how triplet growth does not follow the growth curves of singleton and twins, but after 26 weeks there is a decrease in triplet growth that is about $20 \%$ less than that of singleton. This finding is due to blood flow alteration in uterus and placenta and notably to the restricted intrauterine space that represents also the main cause of preterm labour.

In our study, incidence of caesarean section delivery was $90 \%$ and it was comparable with other studies [13,27]. It is well known that caesarean section triplets have a lower perinatal mortality and morbidity versus vaginally delivered triplets. Triplet vaginal births are associated with higher risk of cord prolapse, foetal collision, reduced placental perfusion and haemorrhage from separating placentae [10]. For these reasons, our gynecologists operated caesarean section for all triplet pregnancies except for two patients who delivered vaginally at 22.3 and 26 weeks respectively. This occurred because the triplets were all extremely low birth weight and their severe immaturity caused the death of the first triplet set and major morbidity of the second one.

Although previous studies have reported a higher neonatal morbidity and mortality in the third born triplet [28], we found no difference in outcome according to the birth order, which was probably due to the policy of delivering all triplet pregnancies by caesarean section.

Several studies compared the perinatal outcome of triplets with twins and singletons matched for gestational age. The most of these studies have not shown significant differences in mortality rates, neonatal complications and survival without significant morbidity, although birth weight was lower in triplets $[13,22,29]$. Thus, the adverse neonatal outcome in multiple births seems to be due primarily to prematurity, as confirmed in our study by the distribution of major morbidity in the categories of lower gestational age. In this contest, it is apparent that efforts aimed at reducing prematurity and avoiding complications should be our primary focus for these patients.

Infants of triplet gestation constitute a significant proportion of NICU admissions [29]. In our study about $3 / 4$ of newborns required intensive care. The neonatal morbidity of higher-order multiples is mainly marked by respiratory problems, occurring in approximately two-thirds of our triplets.

Twelve infants required postnatal transfer because of capacity problems in the primary NICU encountered by the sudden arrival of three or more premature infants. This reflects a critical management of perinatal care due to insufficient availability of intensive therapy beds compared to the incoming demand. As a consequence, premature neonates very often cannot be cared for in the medical center of birth but they have to be transferred to another hospital, which certainly increases risks of death and of long-term sequelae.

It is reported in literature that the risk of cerebral palsy is significantly higher in infants resulting from multiple gestations than from singleton births, and increases exponentially with the number of foetuses [30]. The characteristic of this study limited to the neonatal period does not allow us to obtain data on motor and long-term sequelae. No doubt, it is observed that neurological complications affected $11 \%$ of all infants were affected by neurological complications (including intraventricular hemorrhage or posthemorrhagic hydrocephalus), that could be considered markers of risk for neurologic sequelae.

Despite the small sample, our study adds more information to the limited available studies regarding higher-order gestations and supports the view expressed in the general literature that perinatal mortality in triplet pregnancies have improved in recent years. Well-equipped neonatal units having adequate supportive therapy have decreased the perinatal mortality rate in higher-order multiple pregnancies. In the care of triplet pregnancies, coordination between obstetrical and neonatal staff is essential. Probably this has contributed to the fact that in our sample, although the incidence of prematurity has been $100 \%$, we observed an extremely low mortality rate $(5 \%)$ and relatively low serious associated morbidity.

Nevertheless, maternal and neonatal morbidity in triplet pregnancies remains higher compared with singleton pregnancies. The majority of these pregnancies are conceived by infertility treatments. This demonstrates that the attitude to prevent multiple pregnancies needs to be implemented and that ART should be managed by specialists who not only decide if a pregnancy is possible, but also choose the most appropriate treatment for every infertile couple. Finally, both maternal and neonatal risks should be carefully discussed with these patients when they are counselled.

\section{References}

1. Blondel B, Macfarlane A, Gissler M, Breart G, Zeitlin J, et al. (2006) Preterm birth and multiple pregnancy in European countries participating in the PERISTAT project. BJOG 113: 528-535.

2. Martin JA, Hamilton BE, Sutton PD, Ventura SJ, Mathews TJ, et al. (2010) Births: Final data for 2008. Natl Vital Stat Rep 59: 3-71.

3. Barr S, Poggi S, Keszler M (2003) Triplet morbidity and mortality in a large case series. J Perinatol 23: 368-371.

4. Garg P, Abdel-Latif ME, Bolisetty S, Bajuk B, Vincent T, et al. (2010) Perinata characteristics and outcome of preterm singleton, twin and triplet infants in NSW and the ACT, Australia (1994-2005). Arch Dis Child Fetal Neonatal Ed. 95:F20-F24. 
Citation: Caserta D, Bordi G, Stegagno M, Filippini F, Podagrosi M, et al. (2012) Study of a Population of Triplet Pregnancies: Maternal and Neonatal Outcomes. J Neonatal Bio 1:104. doi:10.4172/2167-0897.1000104

Page 5 of 5

5. Habbema JD, Eijkemans MJ, Nargund G, Beets G, Leridon H, et al. (2009) The effect of in vitro fertilization on birth rates in western countries. Hum Reprod 24: 1414-1419.

6. Kallen B, Finnstrom O, Lindam A, Nilsson E, Nygren KG, et al. (2010) Selected neonatal outcomes in dizygotic twins after IVF versus non-IVF pregnancies. BJOG 117: 676-682.

7. Tandberg A, Bjorge T, Nygard O, Bordahl PE, Skjaerven R (2010) Trends in incidence and mortality for triplets in Norway 1967-2006: the influence of assisted reproductive technologies. BJOG 117: 667-675.

8. American College of Obstetricians and Gynecologists Committee on Practice Bulletins-Obstetrics, Society for Maternal-Fetal Medicine, ACOG Joint Editorial Committee (2004) ACOG Practice Bulletin \#56: Multiple gestation: complicated twin, triplet, and high-order multifetal pregnancy. Obstet Gynecol 104: 869-883.

9. Martin JR, Bromer JG, Sakkas D, Patrizio P (2011) Insurance coverage and in vitro fertilization outcomes: a U.S. perspective. Fertil Steril 95: 964-969.

10. Umeora OU, AneziOkoro EA, Egwuatu VE (2011) Higher-order multiple births in Abakaliki, Southeast Nigeria. Singapore Med J 52: 163-167.

11. Ledger WL, Anumba D, Marlow N, Thomas CM, Wilson EC, et al. (2006) The costs to the NHS of multiple births after IVF treatment in the UK. BJOG 113 21-25.

12. Verberg MF, Macklon NS, Heijnen EM, Fauser BC (2007) ART: iatrogenic multiple pregnancy? Best Pract Res Clin Obstet Gynaecol 21: 129-143.

13. Garite TJ, Clark RH, Elliott JP, Thorp JA (2004) Twins and triplets: the effect of plurality and growth on neonatal outcome compared with singleton infants. Am J Obstet Gynecol 191: 700-707.

14. Royal College of Obstetricians and Gynaecologists (2010) Antenatal Corticosteroids to reduce Neonatal Morbidity and Mortality (Green-top 7). Green-top Guideline No 7.

15. Hall JG (2003) Twinning. Lancet 362: 735-743.

16. Krantz DA, Hallahan TW, He K, Sherwin JE, Evans MI (2011) First-trimester screening in triplets. Am J Obstet Gynecol 205: 364.e1-364.e5.

17. OECD (2011) OECD Family Database, OECD, Paris.
18. Zuppa AA, Scorrano A, Cota F, D'Andrea V, Fracchiolla A, et al. (2007) Neonatal outcomes in triplet pregnancies: assisted reproduction versus spontaneous conception. J Perinat Med 35: 339-343.

19. Keith LG (2001) Higher order multiple gestations. In: Sciarra JJ, ed Gynecol Obstet. Philadelphia: Lippincott and Wilkinson.

20. Al-Suleiman SA, Al-Jama FE, Rahman J, Rahman MS (2006) Obstetric complications and perinatal outcome in triplet pregnancies. $J$ Obstet Gynaecol 26: 200-204.

21. Dudenhausen JW, Maier RF (2010) Perinatal problems in multiple births Dtsch Arztebl Int 107: 663-668.

22. Arlettaz Mieth R, Ersfeld S, Douchet N, Wellmann S, Bucher HU (2011) Higher multiple births in Switzerland: neonatal outcome and evolution over the last 20 years. Swiss Med Wkly 141:w13308.

23. Lamb DJ, Middeldorp CM, van Beijsterveldt CE, Vink JM, Haak MC, et al (2011) Birth weight in a large series of triplets. BMC Pediatr 11:24.

24. Min SJ, Luke B, Min L, Misiunas R, Nugent C, et al. (2004) Birth weight references for triplets. Am J Obstet Gynecol 191: 809-814.

25. Papiernik E (2001) The rate of preterm twin births (22-27 weeks) as a criterion for measuring the quality of prenatal care. Twin Res 4: 426-430.

26. Alexander GR, Kogan M, Martin J, Papiernik E (1998) What are the fetal growth patterns of singletons, twins, and triplets in the United States? Clin Obstet Gynecol 41: 114-125.

27. Nasseri F, Azhir A (2009) The neonatal outcome in twin versus triplet and quadruplet pregnancies. J Res Med Sci 14: 7-12.

28. Ho ML, Chen JY, Ling UP, Chen JH, Huang CM, et al. (1996) Changing epidemiology of triplet pregnancy: etiology and outcome over twelve years. Am J Perinatol 13: 269-275.

29. Ballabh P, Kumari J, AlKouatly HB, Yih M, Arevalo R, et al. (2003) Neonata outcome of triplet versus twin and singleton pregnancies: a matched case control study. Eur J Obstet Gynecol Reprod Biol 107: 28-36.

30. Stanley F, Blair E, Alberman E (2000) The special case of multiple pregnancy. In Cerebral Palsies: Epidemiology and Causal Pathways. Cambridge: Cambridge University Press. 